\title{
Pulmonary fibrosis and its related factors in discharged patients with new coronavirus pneumonia: A cohort study
}

\section{Xiaohe Li}

Shenzhen third people's hospital

\section{Chenguang Shen}

Shenzhen third people's hospital

\section{Lifei Wang}

Shenzhen third people's hospital

\section{Sumit Majumder}

Southern University of Science and Technology

\section{Die Zhang}

Shenzhen third people's hospital

\section{Jamal Deen}

Southern University of Science and Technology

\section{Yanjie Li}

Shenzhen third people's hospital

\section{Ling Qing}

:The Third People's Hospital of Shenzhen

\section{Ying Zhang}

Shenzhen third people's hospital

\section{Chuming Chen}

Shenzhen third people's hospital

\section{Rongrong Zou}

: The Third People's Hospital of Shenzhen

Jianfeng Lan

: The Third People's Hospital of Shenzhen

\section{Ling Huang}

Shenzhen third people's hospital

\section{Cheng Peng}

Shenzhen third people's hospital

\section{Lijiao Zeng}

The Third People's Hospital of Shenzhen

\section{Yanhua Liang}


The Third People's Hospital of Shenzhen

\section{Mengli Cao}

The Third People's Hospital of Shenzhen

\section{Yang Yang}

The Third People's Hospital of Shenzhen

\section{Minghui Yang}

The Third People's Hospital of Shenzhen

\section{Guoyu Tan}

The Third People's Hospital of Shenzhen

\section{Shenghong Tang}

The Third People's Hospital of Shenzhen

\section{Lei Liu}

The Third People's Hospital of Shenzhen

\section{Jing Yuan}

The Third People's Hospital of Shenzhen

\section{Yingxia Liu (D 34762354@qq.com )}

:The Third People's Hospital of Shenzhen

\section{Research}

Keywords: persistent consequences, pulmonary fibrosis, risk factor, COVID-19 recovered patient, lung function

Posted Date: January 26th, 2021

DOI: https://doi.org/10.21203/rs.3.rs-79977/v2

License: (c) (i) This work is licensed under a Creative Commons Attribution 4.0 International License. Read Full License

Version of Record: A version of this preprint was published at Respiratory Research on July 9th, 2021. See the published version at https://doi.org/10.1186/s12931-021-01798-6. 


\section{Abstract}

Background: Thousands of the Coronavirus Disease 2019 (COVID-19) patients have been discharged from hospitals, persistent follow-up studies are required to evaluate the prevalence of post-COVID-19 fibrosis.

Methods: This study involves 462 laboratory confirmed patients with COVID-19 who were admitted to Shenzhen Third People's Hospital from January 11, 2020 to April 26, 2020. A total of 457 patients underwent thin-section chest CT scans during the hospitalization or after discharge to identify the pulmonary lesion. A total of 287 patients were followed up from 90 days to 150 days after the onset of the disease, and lung function tests were conducted in about three months after the onset. The risk factors affecting the persistence of pulmonary fibrosis were identified through regression analysis and the prediction model of the persistence of pulmonary fibrosis was established.

Results: Parenchymal bands, irregular interfaces, reticulation and traction bronchiectasis were the most common CT features in all COVID-19 patients. During the 0-30, 31-60, 61-90, 91-120 and >120 days after onset, $86.87 \%, 74.40 \%, 79.56 \%, 68.12 \%$ and $62.03 \%$ patients developed with pulmonary fibrosis and $4.53 \%, 19.61 \%, 18.02 \%, 38.30 \%$ and $48.98 \%$ patients reversed pulmonary fibrosis, respectively. It was observed that Age, BMI, Fever, and Highest PCT were predictive factors for sustaining fibrosis even after 90 days from onset. A predictive model of the persistence with pulmonary fibrosis was developed basedon the Logistic Regression method with an accuracy, PPV, NPV, Sensitivity and Specificity of the model of $76 \%, 71 \%, 79 \%, 67 \%$, and $82 \%$, respectively. More than half of COVID-19 patients revealed abnormal condition in lung function after 90 days from onset, and the ratio of abnormal lung function did not differ on a statistically significant level between the fibrotic and non-fibrotic groups.

Conclusions: Persistent pulmonary fibrosis was more likely to develop in patients with older age, high $\mathrm{BMI}$, severe/critical condition, fever, long time to turn the viral RNA negative, pre-existing disease and delay to admission. Fibrosis developed in COVID-19 patients could be reversed in about a third of the patients after 120 days from onset. The pulmonary function of less than half of COVID-19 patients could turn to normal condition after three months from onset. An effective prediction model with an average Area Under the Curve (AUC) of 0.84 was established to predict the persistence of pulmonary fibrosis in COVID-19 patients for early diagnosis.

\section{Background}

The Coronavirus Disease 2019 (COVID-19) caused by the severe acute respiratory syndrome coronavirus 2 (SARS-CoV-2) has become a pandemic [1]. As of July 14, 2020, there have been 13,070,095 confirmed cases of COVID-19, including 572,539 deaths globally, posing a serious threat to public health worldwide [2]\{, \#180\}. Infection of SARS-CoV-2 could cause atypical pneumonia with clinical presentation, mainly causing respiratory system infections in humans that can range from a minor common cold to severe diseases $[1,3]$. COVID-19 causes pulmonary syndromes similar to other strains of the coronavirus family, 
namely severe acute respiratory syndrome (SARS) coronavirus and Middle East respiratory syndrome (MERS) coronavirus. Previous data from the study of MERS and SARS suggested that there could be substantial fibrotic consequences following SARS-CoV-2 infection [4, 5].

Chest computed tomography (CT) plays an important role in the diagnosis and treatment of patients with COVID-19 that helps in the diagnosis by depicting lung abnormalities and in the evaluation of the progress of disease and response to treatment [6-8]. Several studies have described the imaging and clinical features of patients during hospitalization or discharged after treatment with COVID-19 using serial thin-section CT [9-14]. At present, it is not known if COVID-19 patients who survived pneumonia would be at risk of chronic sequelae. Persistent follow-up studies are required to evaluate the prevalence of post-COVID-19 fibrosis. In this study, by observing the persistent dynamic changes and the predictors of pulmonary fibrosis in discharged patients with COVID-19, we tried to identify whether the development of pulmonary fibrosis occurs in the survivor population and find early warning indicators that linked to pulmonary fibrosis, with a view to develop an early intervention tool to reduce the occurrence of pulmonary fibrosis.

\section{Methods}

\section{Study Design and Patients}

This study involves 462 patients with COVID-19 who had been admitted to Shenzhen Third People's Hospital from January 11, 2020 to April 26, 2020. COVID-19 patients were diagnosed using quantitative reverse-transcription polymerase chain reaction (qRT-PCR) based on the World Health Organization's interim guidance [15]. Among them, a total of 457 patients underwent thin-section chest CT scans during the hospitalization or after discharge. These patients were discharged from the hospital between January 23, 2020 and May 21, 2020, and the discharge criteria were in line with the Chinese guideline for COVID19 pneumonia. Only one death was included in this study, this patient died 44 days after onset. COVID-19 patients who did not undergo thin-section chest CT scans were excluded in this study.

After discharge, the patients were followed up every four weeks or so, thin-section chest CT scans were performed for each patient at each visit, however, only a part of patients underwent laboratory tests at the follow-up visit because the insufficient medical resources during the pandemic, the final date of follow-up was June 20, 2020. The average observation time of these 457 patients was 80.57 days after onset, the median time was 76 days, including the shortest of 12 days and the longest of 151 days. A total of 289 patients were followed up from 90 days to 150 days after the onset of the disease. During the period of hospitalization, 457 patients underwent routine laboratory tests in every 3 to 7 days, and CT scans in every 3-5 days. In addition, lung function tests were conducted in about 3 months after the onset. Clinical data during hospitalization, and imaging and pulmonary function data after discharge of these patients were obtained from a review of the hospital computer medical system to analyze the pulmonary fibrosis status of COVID-19 patients at different stages. The effect of pulmonary fibrosis on pulmonary function was analyzed simultaneously. The risk factors affecting the persistence of pulmonary fibrosis were 
identified through regression analysis and a prediction model of the persistence with pulmonary fibrosis was established.

\section{CT imaging}

Several non-contrast thin-section chest CT scans were performed for each patient using two independent medical CT machines. Equipment and scanning parameters are as follows: 1) Toshiba TSX-101A64 row spiral CT machine, tube voltage $120 \mathrm{kV}$, automatic tube current, reconstructed layer thickness $1 \mathrm{~mm}$. 2) Shanghai uCT760 64-row spiral CT machine, tube voltage $120 \mathrm{kV}$, automatic tube $40 \mathrm{~mA}$, reconstructed layer thickness $0.625 \mathrm{~mm}$. All image data were observed in the pulmonary window, with window width and window level of $1600 \mathrm{HU}$ and $-550 \mathrm{HU}$, respectively.

\section{Judgment of pulmonary fibrosis and the resolution of pulmonary fibrosis}

All thin-section CT images were independently analyzed by three experienced radiologists to determine the presence of pulmonary fibrosis, and any disagreement was resolved by discussion and consensus. Pulmonary fibrosis on chest CT imaging was defined as a combination of findings including parenchymal bands, irregular interfaces, reticulation and traction bronchiectasis $[5,16,17]$. The Judgment of resolution of pulmonary fibrosis is based on the disappearance of the original fibrosis signs indicated by the patient's lung CT. Patients with resolution are counted repeatedly during each time interval.

\section{Disease severity classification}

Disease severity classification and Murray Score calculation were evaluated as previously reported [18]. Severity of COVID-19 was graded according to China National Health Commission Guidelines for Diagnosis and Treatment of SARS-CoV-2 infection. Laboratory confirmed patients with fever, respiratory manifestations and radiological findings indicative of pneumonia were considered as the moderate cases. Laboratory confirmed patients who met any of the following were considered to have severe COVID-19: (i) respiratory distraction (respiration rate $\geq 30 / \mathrm{min}$; (ii) resting oxygen saturation $\leq 93 \%$, or (iii) arterial oxygen partial pressure (PaO2) / fraction of inspired oxygen (FiO2) $\leq 300 \mathrm{mmHg}(1 \mathrm{mmHg}=$ $0.133 \mathrm{kPa}$ ). Laboratory confirmed patients who had any of the following were considered in critical condition: (i) respiratory failure requiring mechanical ventilation, (ii) shock, or (iii) failure of other organs requiring intensive care unit (ICU).

\section{Statistical Analysis}

Continuous variables were presented as mean \pm standard deviation and categorical variables were presented as $n(\%)$.Continuous variables were presented as mean \pm standard deviation and categorical variables were presented as $n(\%)$. Event frequencies were compared with Chi-squared test, whereas Oneway Analysis of Variance (ANOVA) method was used to compare the continuous parameters between two groups. ANOVA provides a measure of statistical significance ( $p$-value) in the difference between the distributions of a parameter in two groups. A p-value $<0.05$ was considered as significant for all 
statistical tests. Only a subset of statistically significant variables $(p<0.05)$ were considered to develop a simple logistic regression model-based clinical tool for early diagnosis. Variables with $p>0.05$ were not considered for parameter selection and model development. The performance of the model was validated using a five-fold cross validation and assessed with the Receiver Operating Characteristic (ROC) curve, accuracy, Positive Predictive Value (PPV), Negative Predictive Value (NPV), Sensitivity and Specificity. The analysis was performed using a custom-written code in the MATLAB (version R2017b) environment. MATAB is a powerful and commonly used software for mathematical analyses, modeling, classification and prediction.

\section{Results}

\section{Comparison of clinical characteristics between patients with pulmonary fibrosis and with no fibrosis/ resolution of pulmonary fibrosis after 90 days from onset.}

Our study included all 457 confirmed COVID-19 cases admitted to the Shenzhen Third People's Hospital and followed up till June 20,2020 . In order to observe the persistent pulmonary consequences of COVID19 patients, 289 confirmed COVID-19 patients who was followed up more than 90 days after onset were further divided into two groups (group A and group B) according to the progression of pulmonary fibrosis. Group A (GA) had 116 (40.14\%) patients who either had no lung fibrosis, or their lung fibrosis disappeared within 90 days after onset. On the other hand, 173 (59.86\%) patients who still had lung fibrosis after 90 days from onset, were categorized as Group B (GB) (Table 1).

Among the physical characteristics studied, Age and BMI were found to be two significant $(p<0.05)$ risk factors between the two groups. Older patients (mean age 50.68 years vs 33.06 years) and patients with higher BMI (mean BMI $24.1 \mathrm{~kg} / \mathrm{m}^{2}$ vs $22.1 \mathrm{~kg} / \mathrm{m}^{2}$ ) still showed signs of lung fibrosis even after 90 days from onset. Most symptom profiles were comparable between GA and GB. However, a higher proportion of patients in GB had fever compared to GA ( $73 \%$ in GB vs $52 \%$ in GA) that is statistically significant at $\mathrm{p}<0.0001$. In addition, severe/critical COVID patients as well as patients with pre-existing health conditions were observed to be more vulnerable to sustaining lung fibrosis even after 90 days (Table 1).

It can also be seen that, patients in the GB took significantly longer time (21 days vs 14.7 days, $p<$ 0.0001 ) from onset to get virus RNA negative, required longer follow-up period from onset (117.8 days vs 67.8 days, $p<0.0001$ ) and stayed in the hospital for longer period of time (24.4 days vs 19.2 days, $p<$ $0.0001)$. These observations in the temporal patterns can be attributed to the significantly higher number $(29.5 \%$ vs $4.3 \%, p<0.0001)$ of severe/critical COVID cases in GB, since severe/critical COVID patients generally require treatment and follow-up for a longer period time. It is interesting to see that an average delay of $\sim 2$ days before hospital admission following the onset of the symptoms had a significant $(p<$ 0.0001 ) detrimental effect on the patients with pneumonia, thus rendering them more vulnerable to developing and sustaining lung fibrosis for a longer period of time (Table 1) 
Levels of lactic acid, white blood cells (WBC), neutrophils (N), lymphocyte (HB), alanine transaminase (ALT), aspartate aminotransferase (AST), lactate dehydrogenase (LDH), creatine kinase (CK), urea nitrogen (BUN), and D-Dimer did not differ on a statistically significant level $(p>0.05)$ between the two groups (Table 1). However, there were significant differences in some of the laboratory findings between the two groups. These differences (GB vs GA) include lowest oxygenation index (PaO2/FiO2), Lowest CD4 cell, L (lymphocyte), CD4 cells, Highest CRP (C-reactive protein), Highest ESR (erythrocyte sedimentation rate), ESR, Highest lactic acid, Highest PCT, CRP, $\mathrm{Cr}$ (creatinine), Oxygenation index $\left(\mathrm{PaO}_{2} / \mathrm{FiO}_{2}\right), \mathrm{ALB}$ (albumin), PLT (platelet), Highest D-Dimer, Highest IL-6 (interleukin-6), IL-6, and PCT (procalcitonin). It is seen from the lowest oxygenation index $\left(\mathrm{PaO}_{2} / \mathrm{FiO}_{2}\right)$ that the amount of oxygen in the blood drops drastically in patients who sustain fibrosis longer (Table 1).

\section{CT characteristics and dynamic changes in pulmonary fibrosis}

We analyzed the three CT scans for every patient. Ground-glass opacities (GGO), parenchymal bands, irregular interfaces, reticulation and traction bronchiectasis were the most common CT features in all COVID-19 patients. During hospitalization and follow-up, some patients had persistent pulmonary fibrosis (Figure 1), while some patients had resolution of pulmonary fibrosis (Figure 2). Typical CT imaging of a 67-year-old man showed diffuse ground glass opacities in both lungs, and visible parenchymal band in the lower lobe of left lung on initial CT. Diffuse ground glass opacities, consolidation and irregular interfaces with a small amount of pleural effusion were observed on the first follow-up CT. For the third (88 days after symptoms onset) and latest (132 days after symptoms onset) follow-up CT after discharge, diffuse ground glass opacities, consolidation and pleural effusion were still observed in the lungs, although most lesions were resolved (Figure 1). Typical CT imaging findings of a 53-year-old woman showed multiple lesions, a mass of ground glass opacities, consolidation and irregular interfaces on initial CT. The lesions were resolved obviously on the $6^{\text {th }}$ day after onset, further resolved on the $9^{\text {th }}$ and $19^{\text {th }}$ days after onset, and completely resolved in both lungs on $108^{\text {th }}$ day after symptoms onset (Figure 2).

Some patients did not follow-up along with the time extension, a total of 457 patients was included during the 0-30 days after onset in this study. During the 31-60, 61-90, 91-120 and $>120$ days after onset, there were 418, 279, 207 and 79 patients included, respectively, among them, 397 (86.87\%), 311 (74.40\%), 222 (79.57\%), 141 (68.12\%) and 49 (62.03\%) patients developed with pulmonary fibrosis, respectively. It is interesting to see that pulmonary fibrosis developed in COVID-19 patients could be reversed. Resolution of pulmonary fibrosis were found in 18 (4.53\%), 49 (13.61\%), 14 (6.31\%), $30(21.289 \%)$ and $15(30.61 \%)$ COVID-19 patients during the 0-30, 31-60, 61-90, 91-120 and >120 days after onset, respectively (Table 2). There was a total of 397 patients had lung fibrosis, among them, 126 patients had pulmonary fibrosis reversed, the median time of resolution of pulmonary fibrosis was $70.79 \pm 37.67$ days (median: 59 days, range: 8-148 days). In contrast, there were 271 patients who still had pulmonary fibrosis at the last followup. The overall lung fibrosis and overall resolution of fibrosis percentage were $86.87 \%$ and $31.74 \%$, respectively (Table 2). The CT imaging features including ground-glass opacities, meshwork, parenchymal bands, irregular interface and traction bronchiectasis of the COVID-19 patients at different 
stages were analyzed meanwhile. Ground-glass opacities and parenchymal bands are most common CT features in these patients.

\section{Establishment of prediction model of the persistence of pulmonary fibrosis}

A total of 56 features (Table 1) were collected from each patient. After performing the ANOVA, a set of 35 statistically significant $(p<0.05)$ remained for developing the prediction model. However, features related to treatment measures and temporal patterns were not considered for model development since those features are determined by the physicians based on the condition of the patients and thereby are dependent variables.

Among the significant independent variables, it was observed that Age, BMI, Fever, and Highest PCT were predictive factors for sustaining fibrosis even after 90 days (Figure $3 \mathrm{~A}$ ). Among the 289 patients, 288 patients had complete data for these four parameters that were used to develop a predictive model based-on the Logistic regression method. The performance of the model was validated by 5 -fold cross validation and evaluated by the ROC, accuracy, PPV, NPV, Sensitivity and Specificity. Cross validation ensures the consistency of the model's performance while reducing model bias and variance. The confusion matrix of the five-fold cross validation is presented in Figure 4B. An average AUC (Area under the ROC Curve) of 0.84 obtained from 5 -fold cross validation that affirms a good reliability of the predictive model. The accuracy, PPV, NPV, Sensitivity and Specificity of the model was $76 \%, 71 \%, 79 \%$, $67 \%$, and $82 \%$, respectively (Figure 4 C).

\section{Pulmonary function of patients with pulmonary fibrosis and with no fibrosis/ resolution of pulmonary fibrosis after 90 days from onset.}

In order to observe the effect of pulmonary fibrosis on lung function, 33 patients from Group $A(G A)$ and 114 patients from Group B (GB) underwent pulmonary function testing after 90 days from onset. Six patients $(18.18 \%)$ in GA and 26 patients $(22.81 \%)$ in GB had a pulmonary function abnormality. Two patients (6.06\%) in GA and 5 patients (4.39\%) in GB were diagnosed with obstructive pulmonary disorder. Restrictive disorder was found in 1 patient $(3.03 \%)$ in GA and 8 patients $(7.02 \%)$ in GB. In addition, 3 patients $(9.09 \%)$ in GA and 13 patients $(11.40 \%)$ in GB had small airway disorder. MEF 25 decrease was found in 12 patients (36.36\%) in GA and 46 patients (40.35\%) patients in GB. 2 patients (6.06\%) in GA and 8 patients $(7.02 \%)$ in GB had a pulmonary diffusion abnormality (Table 3). Overall, 18 patients $(55.55 \%)$ and 76 patients $(66.67 \%)$ revealed abnormal condition in pulmonary function test. It is interesting to see that the ratio of abnormal lung function, obstructive disorder, restrictive disorder, small airway disorder, MEF 25 decrease, abnormal lung diffusion and overall abnormalities in lung function test did not differ on a statistically significant level $(p>0.05)$ between the two groups.

\section{Discussion}

Data from previous coronavirus infections such as SARS, as well as emerging data from the COVID-19 pandemic, suggest there could be substantial fibrotic consequences following SARS-CoV-2 infection [5, 
19-22]. Given the huge numbers of individuals infected by SARS-CoV-2, it is important to identify and predict the occurrence of pulmonary fibrosis in the survivor population after discharge.

In our study, thin-section CT scans obtained in hospitalized or discharged patients have shown that fibrosis occurred in a large proportion of the COVID-19 patients, and it occurred in more than a half even for the subset of patients after 120 days from onset. Numerous similarities were found among SARS-CoV coronavirus, MERS coronavirus and SARS-CoV-2. In an early follow-up study of patients with SARS, $62 \%$ patients revealed CT evidence of pulmonary fibrosis at a mean follow-up duration of 37 days after hospital discharge [5], whereas in a follow-up study of patients with MERS, 33\% had radiographic evidence of pulmonary fibrosis [4]. In view of this, the infection of SARS-CoV-2 has a high incidence of pulmonary fibrosis, which is comparable to those of SARS and MERS.

Clinically, patients with fibrosis after long term from onset were significantly older, with higher BMI and with significantly higher proportions of fever and severe/critical COVID cases than those without fibrosis. These results implied that fibrosis was likely to be more common in elderly, obese and severe/critical patients, similar to those patients with SARS [5], and COVID-19 patients with fever are more vulnerable to developing pulmonary fibrosis. In addition, patients with pulmonary fibrosis need a much longer time to turn the RNA of SARS-CoV-2 negative in their bodies, implied that the longer the virus lived in vivo, the more damage it did to the patient's lungs. Meanwhile, it is interesting to observe that the delay to admission following the onset of the symptoms had a significant detrimental effect on the patients with pneumonia, suggested that it is important to seek medical advice in time after onset for the COVID-19 patients.

The pulmonary fibrosis of COVID-19 patients in our study was identified by reviewing the CT scans, in many previous studies, this method was also used to diagnose the lung damage resulted from viral pneumonia, such as lung fibrosis $[4,9,14,22,23]$. Thin-section CT scans from all the patients showed that evidence of fibrosis was found in almost a half of patients, which was consistent with the findings of some previous studies in COVID-19 patients [9, 14,24]. We found that pulmonary fibrosis developed in COVID-19 patients could be reversed in a part of population. The pulmonary fibrosis in some patients was persistent during the follow-up period, it is necessary to follow-up on these patients for longer time to assess the persistent pulmonary consequence of them.

For the survivors from SARS, at one-year follow-up, pulmonary function testing showed a pulmonary function abnormality in a subset of patients, who suffered with poor quality of life after discharge [25]. In this study, the infection of SARS-CoV-2 has a high incidence of pulmonary fibrosis, however, it is interesting to see that about half of COVID-19 patients suffered with abnormal lung function after 90 days from onset, and the ratio of abnormal lung function did not differ on a statistically significant level between the fibrotic and non-fibrotic groups. These results suggested that the pulmonary function of less than half of COVID-19 patients with or without pulmonary fibrosis could turn to normal condition after 3 months from onset. It is reassuring to see that patients with COVID-19 seem to have a better prognosis than those with severe acute respiratory syndrome coronavirus. 
Significantly, we established a prediction model for the prediction of the persistence of pulmonary fibrosis in this study for early diagnosis. It was observed that Age, BMI, Fever, and Highest PCT were predictive factors for sustaining fibrosis even after 90 days. The patients with high risk to develop persistent pulmonary fibrosis deserve special attention, anti-fibrosis drugs can be considered in the early stage of treatment to prevent further damage to the lungs of such kind of patients.

This study has some limitations. First, the follow-up time for these patients is not long enough, and it is unknown whether the pulmonary fibrosis will permanently remain. Second, there is no histologic confirmation of fibrosis in any of the patients, although the signs on thin-section CT scans are convincing. Third, COVID-19 patients admitted to our hospital in this study were mostly imported from abroad or from outside the province, most patients were non-residents of Shenzhen, most patients left Shenzhen after their condition improved, so the lost rate of follow up in this study is high, a certain part of discharged patients were lost, only 79 patients are included during $>120$ days after onset. Fourth, this study is centered mainly on the development of lung fibrosis lesions and on their resolution. However, it did not take into due consideration important clinical parameters to confirm that CT images. Forth, the follow up did not take in due consideration the levels of urinary hydroxyproline (which derive from the collagen catabolism) obtained from urine analysis, that may demonstrate the true existence an increased lung collagen resorption during the fibrosis resolution.

\section{Conclusions}

Persistent pulmonary fibrosis was more likely to develop in patients with older age, high BMI, severe/critical condition, fever, long time to turn the viral RNA negative in vivo, pre-existing disease and delay to admission. Pulmonary fibrosis occurred in a large proportion of the COVID-19 patients, even for the patients after 120 days from onset, the fibrosis developed in COVID-19 patients could be reversed in about a third of the patients after 120 days from onset. The pulmonary function of less than half of COVID-19 patients with pulmonary fibrosis could turn to normal condition after 3 months from onset. An effective prediction model with an average AUC of 0.84 was established to predict the persistence of pulmonary fibrosis in COVID-19 patients for early diagnosis. By doing this, we hope to deliver appropriate clinical care and in time design interventional trials to the patients with high risk to develop persistent pulmonary fibrosis. Future follow-up studies with a longer follow-up period would be necessary in order to confirm our findings and better determine the long-term outcomes of patients who recovered from COVID19.

\section{List Of Abbreviations}

COVID-19: The Coronavirus Disease 2019; SARS: Severe acute respiratory syndrome; MERS: coronavirus and Middle East respiratory syndrome; CT: Chest computed tomography; qRT-PCR: quantitative reversetranscription polymerase chain reaction; ROC: Receiver Operating Characteristic; PPV: Positive Predictive Value; NPV: Negative Predictive Value; AUC: Area under the ROC Curve. 


\section{Declarations}

\section{Ethics approval and consent to participate}

This study was conducted at Shenzhen Third People's Hospital and approved by the Ethics Committees, each patient gave written informed consent.

\section{Consent for publication}

Not applicable.

\section{Availability of data and materials}

The datasets used and/or analyzed during the current study are available from the corresponding author on reasonable request.

\section{Competing interests}

The authors declare no competing interests.

\section{Funding}

This study was supported by Shenzhen Science and Technology Research and Development Project (202002073000001 and 202002073000002), National Natural Science Foundation of China (81902058), Shenzhen Science and Technology Research and Development Project (202002073000002), Shenzhen Fund for Guangdong Provincial High-level Clinical Key Specialties (SZGSP011), Sanming Project of Medicine in Shenzhen (SZSM201512005). SM and MJD acknowledge the Canada Research Chair Program for partial support of their work.

\section{Authors' contributions}

YL, LL, JY and XL designed this study; XL, CS, LH, DZ, YL, LQ, YZ, CC, RZ, JL, CF, LZ, YL, MC, YY, MY, GT, ST collected the data; $Y L, X L, S M, J D, C S$ analyzed and interpreted the data; Predictions and discussions: $\mathrm{XL}, \mathrm{SM}, \mathrm{JD}, \mathrm{CS}$ established the prediction model; XL, CS, SM and JD wrote the manuscript. All authors read and approved the final manuscript.

\section{Acknowledgement}

Not applicable.

\section{References}

1. Huang C, Wang Y, Li X, Ren L, Zhao J, Hu Y, Zhang L, Fan G, Xu J, Gu X, Cheng Z, Yu T, Xia J, Wei Y, Wu W, Xie X, Yin W, Li H, Liu M, Xiao Y, Gao H, Guo L, Xie J, Wang G, Jiang R, Gao Z, Jin Q, Wang J, 
Cao B. Clinical features of patients infected with 2019 novel coronavirus in Wuhan, China. Lancet. 2020;395(10223):497-506.

2. https://covid19.who.int/. Data last updated: 2020/7/14, 10:42am CEST.

3. Rothan HA, Byrareddy SN. The epidemiology and pathogenesis of coronavirus disease (COVID-19) outbreak. J Autoimmun. 2020;109:102433.

4. Das KM, Lee EY, Singh R, Enani MA, Al Dossari K, Van Gorkom K, Larsson SG, Langer RD. Follow-up chest radiographic findings in patients with MERS-CoV after recovery. Indian J Radiol Imaging. 2017;27(3):342-9.

5. Antonio GE, Wong KT, Hui DS, Wu A, Lee N, Yuen EH, Leung CB, Rainer TH, Cameron P, Chung SS, Sung JJ, Ahuja AT. Thin-section CT in patients with severe acute respiratory syndrome following hospital discharge: preliminary experience. Radiology. 2003;228(3):810-5.

6. Rorat M, Zińczuk A, Szymański W, Simon K, Guziński M. Usefulness of portable chest radiography in initial diagnosis of COVID-19. Pol Arch Intern Med. 2020.

7. Majidi H, Bani-Mostafavi ES, Mardanshahi Z, Godazandeh F, Ghasemian R, Heydari K, AlizadehNavaei R. High-resolution computed tomography finding in 552 patients with symptomatic COVID19: first report from north of Iran. Emerg Radiol. 2020:1-7.

8. Adams HJA, Kwee TC, Yakar D, Hope MD, Kwee RM. Chest CT Imaging Signature of Coronavirus Disease 2019 Infection: In Pursuit of the Scientific Evidence. Chest. 2020.

9. Xu X, Yu C, Qu J, Zhang L, Jiang S, Huang D, Chen B, Zhang Z, Guan W, Ling Z, Jiang R, Hu T, Ding Y, Lin L, Gan Q, Luo L, Tang X, Liu J. Imaging and clinical features of patients with 2019 novel coronavirus SARS-CoV-2. Eur J Nucl Med Mol Imaging. 2020;47(5):1275-80.

10. Ding X, Xu J, Zhou J, Long Q. Chest CT findings of COVID-19 pneumonia by duration of symptoms. Eur J Radiol. 2020;127:109009.

11. Shi H, Han X, Zheng C. Evolution of CT Manifestations in a Patient Recovered from 2019 Novel Coronavirus (2019-nCoV) Pneumonia in Wuhan, China. Radiology. 2020;295(1):20.

12. Pan F, Ye T, Sun P, Gui S, Liang B, Li L, Zheng D, Wang J, Hesketh RL, Yang L, Zheng C. Time Course of Lung Changes at Chest CT during Recovery from Coronavirus Disease 2019 (COVID-19). Radiology. 2020;295(3):715-21.

13. Chung M, Bernheim A, Mei X, Zhang N, Huang M, Zeng X, Cui J, Xu W, Yang Y, Fayad ZA, Jacobi A, Li K, Li S, Shan H. CT Imaging Features of 2019 Novel Coronavirus (2019-nCoV). Radiology. 2020;295(1):202-7.

14. Yu M, Liu Y, Xu D, Zhang R, Lan L, Xu H. Prediction of the Development of Pulmonary Fibrosis Using Serial Thin-Section CT and Clinical Features in Patients Discharged after Treatment for COVID-19 Pneumonia. Korean J Radiol. 2020;21(6):746-55.

15. Bernheim A, Mei X, Huang M, Yang Y, Fayad ZA, Zhang N. Chest CT Findings in Coronavirus Disease19 (COVID-19): Relationship to Duration of Infection. Radiology. 2020;295(3):200463. 
16. Zerhouni EA, Naidich DP, Stitik FP, Khouri NF, Siegelman SS. Computed tomography of the pulmonary parenchyma. Part 2: Interstitial disease. J Thorac Imaging. 1985;1(1):54-64.

17. Raghu G, Remy-Jardin M, Myers JL, Richeldi L, Ryerson CJ, Lederer DJ, Behr J, Cottin V, Danoff SK, Morell F, Flaherty KR, Wells A, Martinez FJ, Azuma A, Bice TJ, Bouros D, Brown KK, Collard HR, Duggal A, Galvin L, Inoue Y, Jenkins RG, Johkoh T, Kazerooni EA, Kitaichi M, Knight SL, Mansour G, Nicholson AG, Pipavath SNJ, Buendía-Roldán I, Selman M, Travis WD, Walsh S, Wilson KC. Diagnosis of Idiopathic Pulmonary Fibrosis. An Official ATS/ERS/JRS/ALAT Clinical Practice Guideline. Am J Respir Crit Care Med. 2018;198(5):e44-e68.

18. Yang Y, Shen C, Li J, Yuan J, Wei J, Huang F, Wang F, Li G, Li Y, Xing L, Peng L, Yang M, Cao M, Zheng H, Wu W, Zou R, Li D, Xu Z, Wang H, Zhang M, Zhang Z, Gao GF, Jiang C, Liu L, Liu Y. Plasma IP-10 and MCP-3 levels are highly associated with disease severity and predict the progression of COVID19. J Allergy Clin Immunol. 2020;146(1):119-27.e4.

19. Rajasurya V, Gunasekaran K, Damarla V, Kolluru A. A Fatal Case of Coronavirus Disease 2019 (COVID-19) in a Patient With Idiopathic Pulmonary Fibrosis. Cureus. 2020;12(6):e8432.

20. Gold JAW, Wong KK, Szablewski CM, Patel PR, Rossow J, da Silva J, Natarajan P, Morris SB, Fanfair RN, Rogers-Brown J, Bruce BB, Browning SD, Hernandez-Romieu AC, Furukawa NW, Kang M, Evans ME, Oosmanally N, Tobin-D'Angelo M, Drenzek C, Murphy DJ, Hollberg J, Blum JM, Jansen R, Wright DW, Sewell WM, 3rd, Owens JD, Lefkove B, Brown FW, Burton DC, Uyeki TM, Bialek SR, Jackson BR. Characteristics and Clinical Outcomes of Adult Patients Hospitalized with COVID-19 - Georgia, March 2020. MMWR Morb Mortal Wkly Rep. 2020;69(18):545-50.

21. Wang J, Wang BJ, Yang JC, Wang MY, Chen C, Luo GX, He WF. [Advances in the research of mechanism of pulmonary fibrosis induced by Corona Virus Disease 2019 and the corresponding therapeutic measures]. Zhonghua Shao Shang Za Zhi. 2020;36(0):E006.

22. Xie L, Liu Y, Xiao Y, Tian Q, Fan B, Zhao H, Chen W. Follow-up study on pulmonary function and lung radiographic changes in rehabilitating severe acute respiratory syndrome patients after discharge. Chest. 2005;127(6):2119-24.

23. Bernheim A, Mei X, Huang M, Yang Y, Fayad ZA, Zhang N, Diao K, Lin B, Zhu X, Li K, Li S, Shan H, Jacobi A, Chung M. Chest CT Findings in Coronavirus Disease-19 (COVID-19): Relationship to Duration of Infection. Radiology. 2020;295(3):200463.

24. Zhu J, Zhong Z, Li H, Ji P, Pang J, Li B, Zhang J. CT imaging features of 4121 patients with COVID19: A meta-analysis. J Med Virol. 2020;92(7):891-902.

25. Gentile F, Aimo A, Forfori F, Catapano G, Clemente A, Cademartiri F, Emdin M, Giannoni A. COVID-19 and risk of pulmonary fibrosis: the importance of planning ahead. Eur J Prev Cardiol. 2020:2047487320932695.

\section{Tables}


Table. 1 Epidemiological and baseline clinical features of 289 patients who were followed up from 90 days to 150 days after the onset. 


$\begin{array}{ccc}\mathrm{a}_{\text {Group A }} & \mathrm{b}_{\text {Group B }} & \text { P value } \\ (\mathrm{N}=116) & (\mathrm{N}=173)\end{array}$

Physical characteristics

$\begin{array}{lrrr}\text { Gender (male ratio, \%) } & 44 \% & 52 \% & 0.2212 \\ \text { Age (year) } & 33.06 \pm 17.50 & 50.68 \pm 13.25 & <0.0001 \\ \text { BMI }\left(\mathrm{kg} / \mathrm{m}^{2}\right) & 22.10 \pm 3.43 & 24.06 \pm 3.21 & <0.0001\end{array}$

Pre-existing Conditions (with pre-existing disease ratio)

Pre-existing disease (\%)

DM (\%)

Hypertension (\%)

Coronary heart disease (\%)

Respiratory disease (\%)

\section{Others}

Case type: (severe/critical ratio, \%)

Time from onset to virus RNA negative (day)

Time from onset to admission (day)

Length of hospitalization (day)

Follow-up period from onset (day)

\section{Symptoms}

Fever (\%)

Fatigue (\%)

Dry cough (\%)

Cough (\%)

Expectoration (\%)

Diarrhea (\%)
$19.0 \%$

$0 \%$

$3.5 \%$

$3.5 \%$

$3.5 \%$

$4.3 \%$

$14.72 \pm 8.22$

$3.01 \pm 3.03$

$19.17 \pm 6.85$

$67.78 \pm 36.09$
$38.2 \% \quad 0.0008$

$7.5 \% \quad 0.0063$

$19.1 \% \quad 0.0002$

$6.9 \% \quad 0.3131$

$5.2 \% \quad 0.6776$

$\begin{array}{rrr}4.3 \% & 29.5 \% & <0.0001 \\ 14.72 \pm 8.22 & 20.99 \pm 9.34 & <0.0001 \\ 3.01 \pm 3.03 & 5.22 \pm 4.29 & <0.0001 \\ 19.17 \pm 6.85 & 24.35 \pm 9.70 & <0.0001 \\ 67.78 \pm 36.09 & 117.77 \pm 14.24 & <0.0001\end{array}$




\section{Laboratory findings}

Oxygenation index $\left(\mathrm{PaO}_{2} / \mathrm{FiO}_{2}\right)$

Lactic acid (mmol/L)

WBC $\left(10^{9} / \mathrm{L}\right)$

$\mathrm{N}\left(10^{9} / \mathrm{L}\right)$

$\mathrm{L}\left(10^{9} / \mathrm{L}\right)$

HB $(g / L)$

$\operatorname{PLT}\left(10^{9} / \mathrm{L}\right)$

ALB (g/L)

ALT (U/L)

AST (U/L)

$\mathrm{LDH}(\mathrm{U} / \mathrm{L})$

CK (U/L)

BUN (mmol/L)

$\mathrm{Cr}(\mu \mathrm{mol} / \mathrm{L})$

ESR $(\mathrm{mm} / \mathrm{h})$

D-Dimer $(\mu \mathrm{g} / \mathrm{mL})$

CRP (mg/dL)

IL-6 (ng/L)

PCT $(\mathrm{ng} / \mathrm{mL})$

CD4 cells (count $/ \mu \mathrm{L}$ )

Highest CRP (mg/dL)

Highest IL-6 (ng/L)

Highest PCT (ng/mL)

Lowest CD4 cell (count $/ \mu \mathrm{L}$ )

Highest ESR $(\mathrm{mm} / \mathrm{h})$

Highest D-Dimer $(\mu \mathrm{g} / \mathrm{mL})$

Highest lactic acid (mmol/L)

Lowest oxygenation index $\left(\mathrm{PaO}_{2} / \mathrm{FiO}_{2}\right)$
$463.15 \pm 123.05$

$1.29 \pm 0.45$

$5.179 \pm 2.04$

$2.85 \pm 1.47$

$1.7659 \pm 0.88$

$137.11 \pm 15.43$

$210.35 \pm 65.32$

$43.91 \pm 3.18$

$24.54 \pm 26.74$

$28.34 \pm 20.18$

$267.37 \pm 144.00$

$115.30 \pm 194.97$

$4.16 \pm 1.41$

$60.92 \pm 17.81$

$23.01 \pm 20.32$

$0.42 \pm 0.37$

$12.25 \pm 25.58$

$11.27 \pm 19.57$

$0.12 \pm 0.10$

$771.89 \pm 419.79$

$19.23 \pm 30.71$

$8.91 \pm 11.92$

$0.29 \pm 0.09$

$700.81 \pm 383.69$

$32.00 \pm 29.07$

$0.63 \pm 0.84$

$2.19 \pm 0.93$

$4.09 \pm 1.62$

$68.75 \pm 20.66$

$35.95+23.00$

$0.52 \pm 0.68$

0.1732

$23.96 \pm 30.38$

0.0008

$20.68 \pm 30.70$

0.0112

$0.09 \pm 0.08$

0.0053

$535.68 \pm 276.76<0.0001$

$47.81 \pm 54.94<0.0001$

$45.62 \pm 142.25 \quad 0.0099$

$0.32 \pm 0.08 \quad 0.0003$

$471.46 \pm 274.76<0.0001$

$50.29 \pm 30.70<0.0001$

$1.40 \pm 3.11 \quad 0.0095$

$2.62 \pm 0.85 \quad 0.0001$

$379.97 \pm 118.46 \quad 270.93 \pm 124.19<0.0001$ 
a Patients who either had no lung fibrosis, or their lung fibrosis disappeared within 90 days after onset.

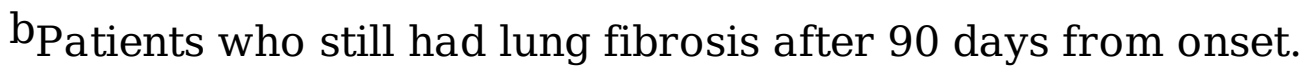

Abbreviations: $\mathrm{BMI}$, body mass index; $\mathrm{DM}$, diabetes mellitus; $\mathrm{PaO}_{2}$, partial pressure of oxygen; $\mathrm{FIO}_{2}$, fraction of inspired oxygen; WBC, white blood cells; N, neutrophils; L, lymphocyte; HB, hemoglobin; PLT, platelet; ALB, albumin; ALT, alanine transaminase; AST, aspartate aminotransferase; LDH, lactate dehydrogenase; $\mathrm{CK}$, creatine kinase; $\mathrm{BUN}$, urea nitrogen; Cr, creatinine; ESR, erythrocyte sedimentation rate; CRP, C-reactive protein; IL-6, interleukin-6; PCT, procalcitonin.

Table 2. Dynamic changes of pulmonary fibrosis in 457 patients at different stages after onset of COVID-19 


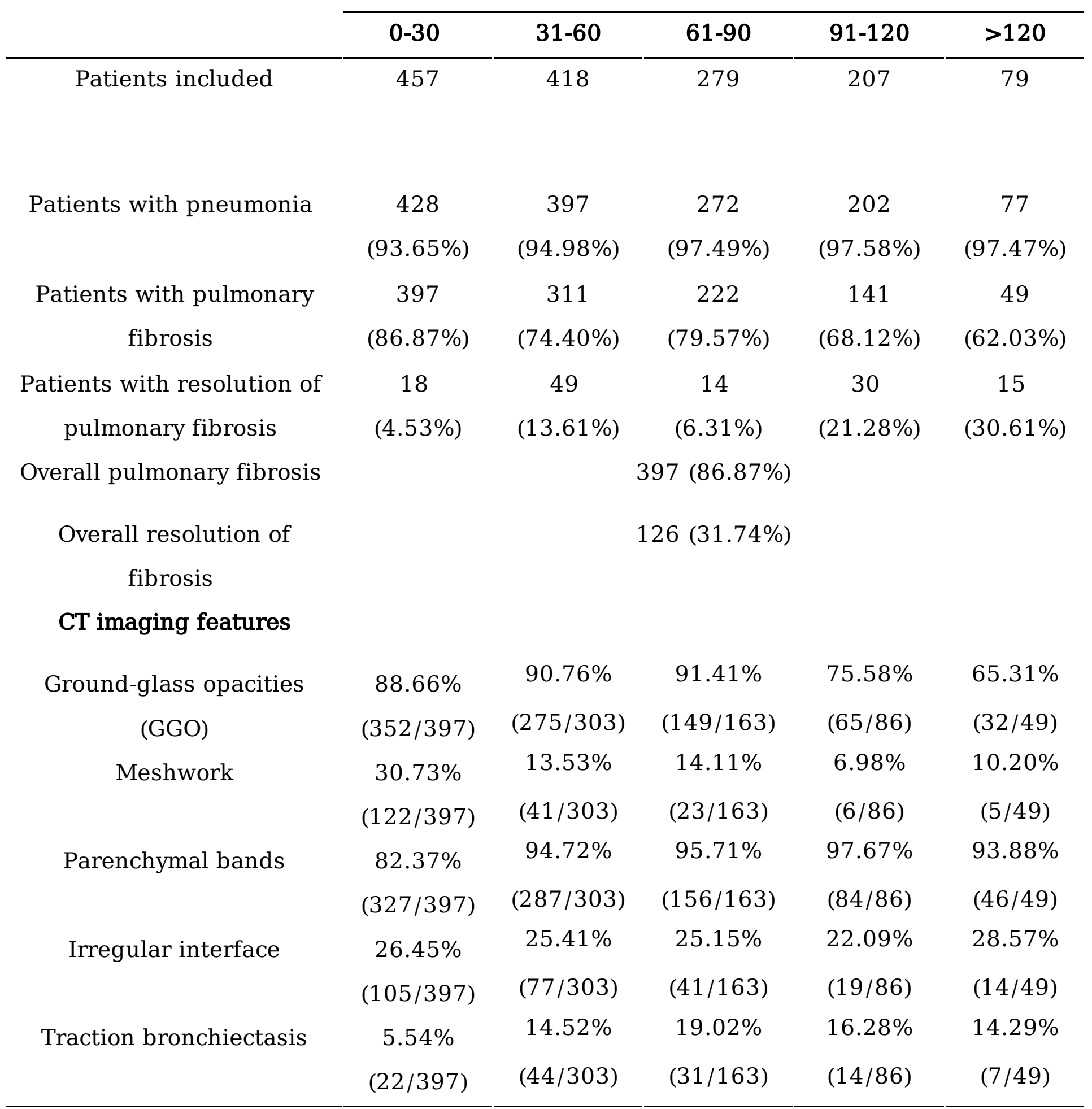

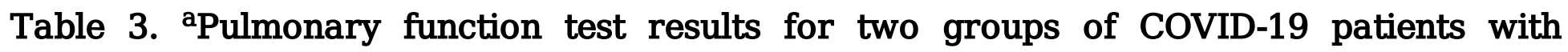
pulmonary fibrosis. 


\begin{tabular}{|c|c|c|c|}
\hline & $\begin{array}{c}\mathrm{b}_{\text {Group A }} \\
(\mathrm{N}=33)\end{array}$ & $\begin{array}{c}\mathrm{C}_{\mathrm{Group}} \mathrm{B} \\
\mathrm{aN}=114 \square\end{array}$ & $\begin{array}{c}P \\
\text { value }\end{array}$ \\
\hline \multirow[t]{2}{*}{$\mathrm{d}_{\mathrm{Abnormal}}$ lung function ratio } & $18.18 \%(6 / 33)$ & $22.81 \%$ & 0.7433 \\
\hline & & $(26 / 114 \square$ & \\
\hline Obstructive disorder ratio & $6.06 \%(2 / 33)$ & $4.39 \%(5 / 114)$ & 0.9471 \\
\hline Restrictive disorder ratio & $3.03 \%(1 / 33)$ & $7.02 \%(8 / 114)$ & 0.6679 \\
\hline \multirow[t]{2}{*}{ Small airway disorder ratio } & $9.09 \%(3 / 33)$ & $11.40 \%$ & 0.9535 \\
\hline & & $(13 / 114)$ & \\
\hline \multirow[t]{2}{*}{$\mathrm{e}_{\mathrm{MEF}} 25$ decrease } & $36.36 \%$ & $40.35 \%$ & 0.8333 \\
\hline & $(12 / 33)$ & $(46 / 114)$ & \\
\hline Abnormal lung diffusion function & $6.06 \%(2 / 33)$ & $7.02 \%(8 / 114)$ & 0.8413 \\
\hline Overall abnormalities ratio in pulmonary function & $55.55 \%$ & $66.67(76 / 114)$ & 0.8787 \\
\hline test & $(18 / 33)$ & & \\
\hline
\end{tabular}

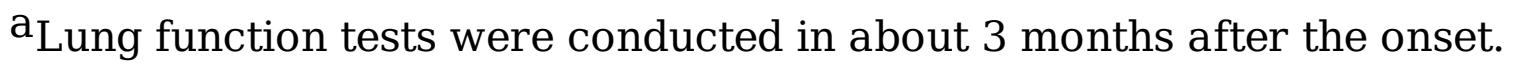

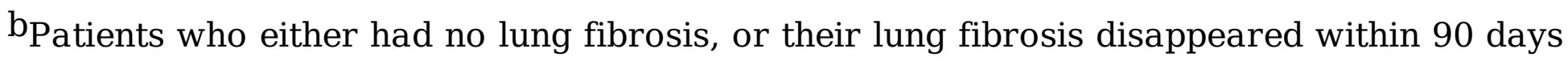
after onset.

CPatients who still had lung fibrosis after 90 days from onset.

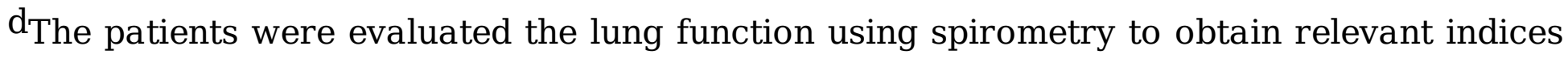
including first second exhalation volume (FEV1), forced vital capacity (FVC), FEV1/FVC\%, forced expiratory flow rate at $50 \%$ and $75 \%$ of FVC (FEF50\%, FEF75\%), forced expired flow at $25-75 \%$ of FVC (FEF25-75\%), inspiratory reserve volume (IRV), tidal volume (TV) and expiratory reserve volume (ERV).

e MEF 25, maximal expiratory flow after $25 \%$ of the forced vital capacity has not been exhaled.

\section{Figures}




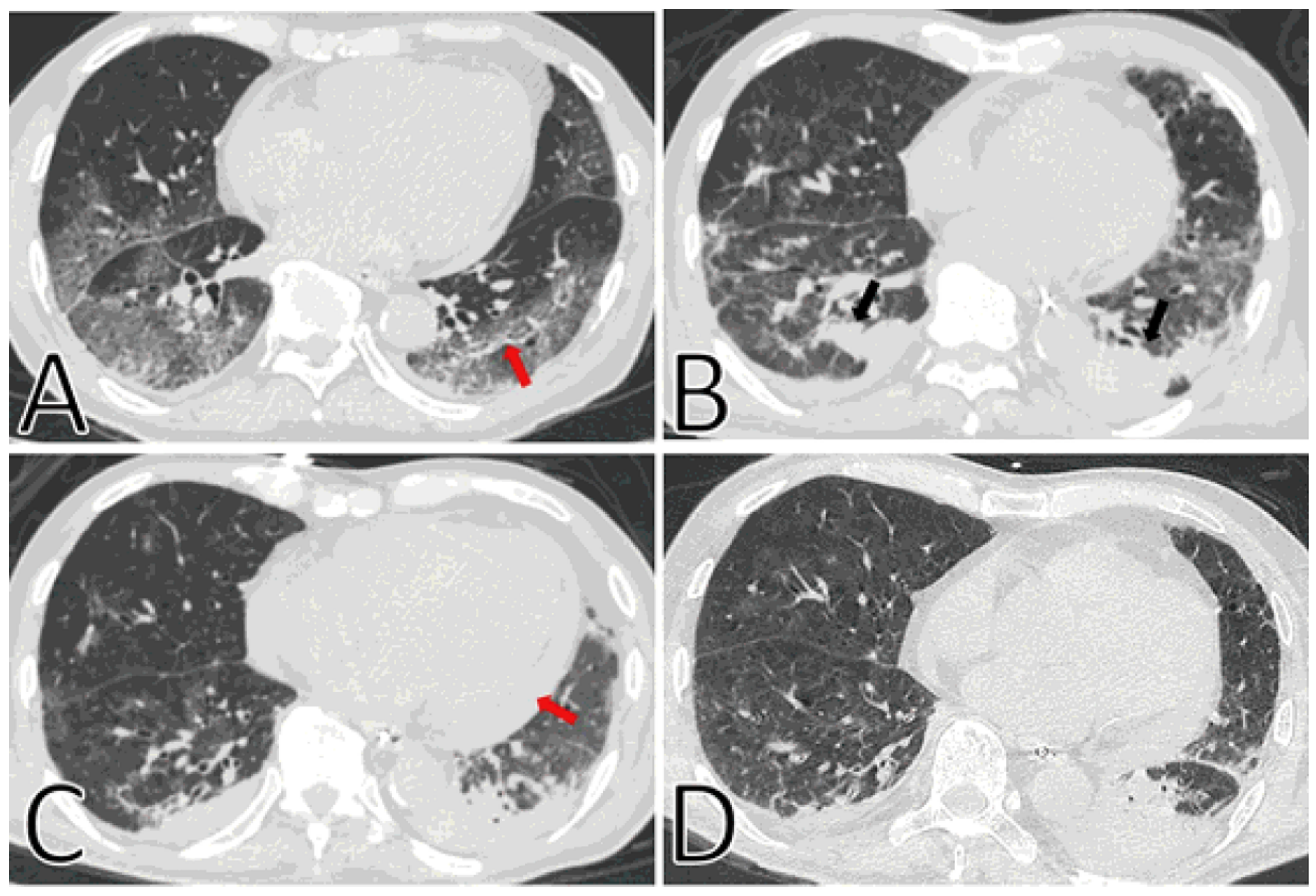

Figure 1

Typical CT imaging findings of a 67-year-old man with persistent pulmonary fibrosis. A. Thin-section chest CT scan in our hospital on January 26, 2020 (9 days after symptoms onset). Chest CT imaging showed diffuse ground glass opacities in both lungs, and visible parenchymal band in the lower lobe of left lung (red arrow). B. On March 23, 2020 (66 days after symptoms onset), diffuse ground glass opacities were absorbed partially in both lungs and new consolidation was observed. Irregular interfaces (black arrows) with a small amount of pleural effusion were observed in the lower lobes of both lungs. C. On April 14, 2020 (88 days after symptoms onset), diffuse ground glass opacities in both lungs were further absorbed, and the consolidation in both lower lungs was also absorbed. Besides, a small amount of bilateral pleural effusion and new pericardial effusion were observed (red arrow). D. On May 28, 2020 (132 days after symptoms onset), a little ground glass opacities could still be observed in both lungs, consolidation was seen in the lower lobe of both lungs, which was obvious in the lower lobe of the left lung. Besides, there was still a small amount of pleural effusion in the left pleural cavity. 


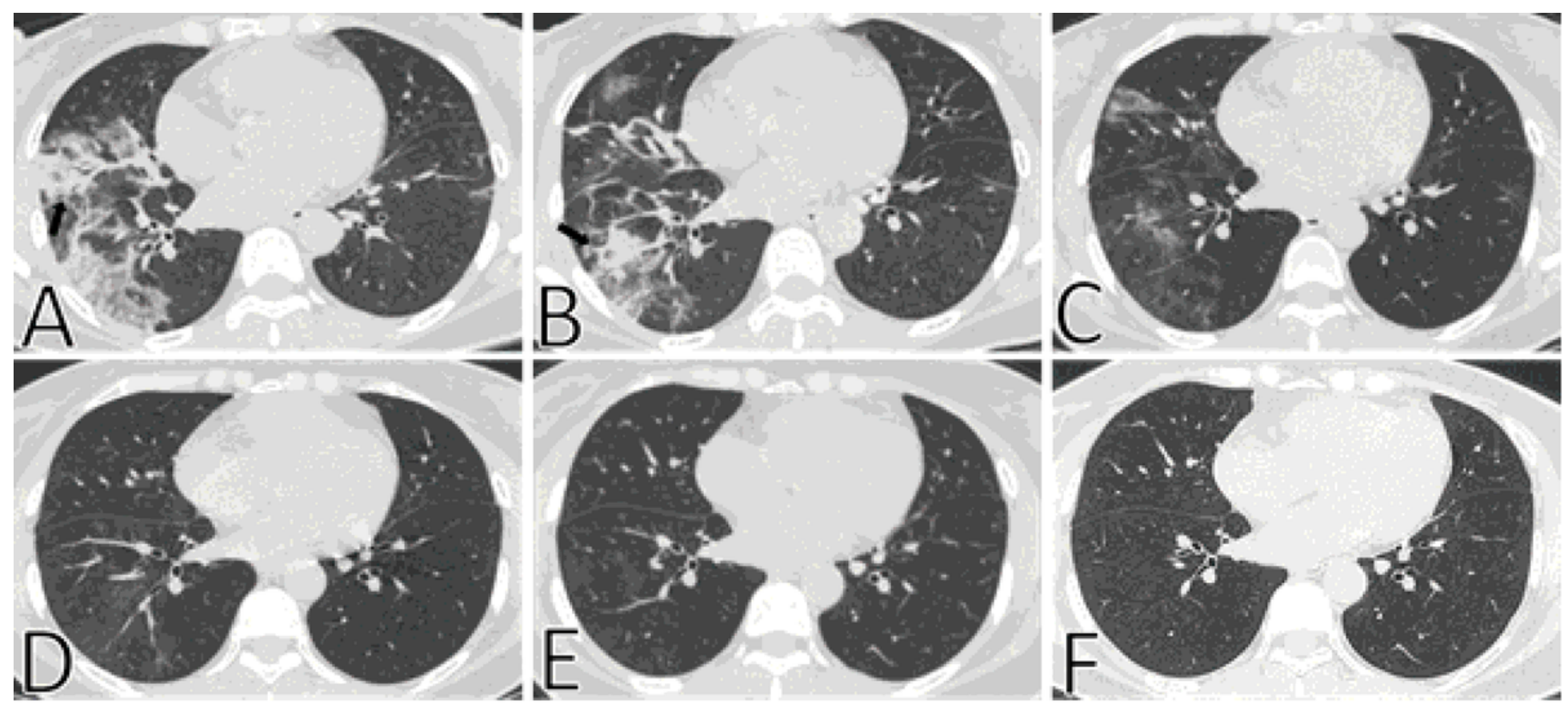

\section{Figure 2}

Typical CT imaging findings of a 53-year-old woman with absorbed pulmonary fibrosis. A. Thin-section chest CT scan in our hospital on February 7, 2020 (4 days after symptoms onset). Chest CT imaging showed multiple lesions in both lungs, a mass of ground glass opacities were observed in the middle and lower lobes of the right lung, with consolidation and irregular interfaces (black arrow). B. On February 9 , 2020 (6 days after symptoms onset), the absorption of the lesion was obvious, ground glass opacities, consolidation and irregular interfaces (black arrow) were still observed. C. On February 12, 2020 (9 days after symptoms onset), the lung lesions were further absorbed, the density of consolidation decreased. D and E. On February 22, 2020 (19 days after symptoms onset) and March 14, 2020 (40 days after symptoms onset), respectively, only a little ground glass opacities were observed in the lower lobe of the right lung, with obscure boundaries. F. On May 22, 2020 (108 days after symptoms onset), the lesions in both lungs have been completely absorbed. 
A

\begin{tabular}{|c|c|}
\hline Parameters & $\boldsymbol{P}$ \\
\hline Age & $<0.00001$ \\
\hline BMI & $<0.034$ \\
\hline Fever & $\mathbf{0 . 0 5 5}$ \\
\hline Highest PCT & $<\mathbf{0 . 0 0 2}$ \\
\hline
\end{tabular}

C

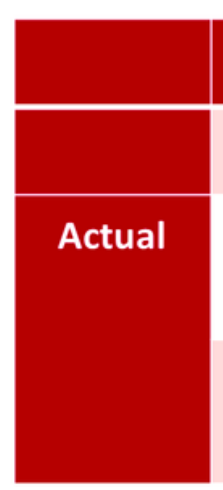

B

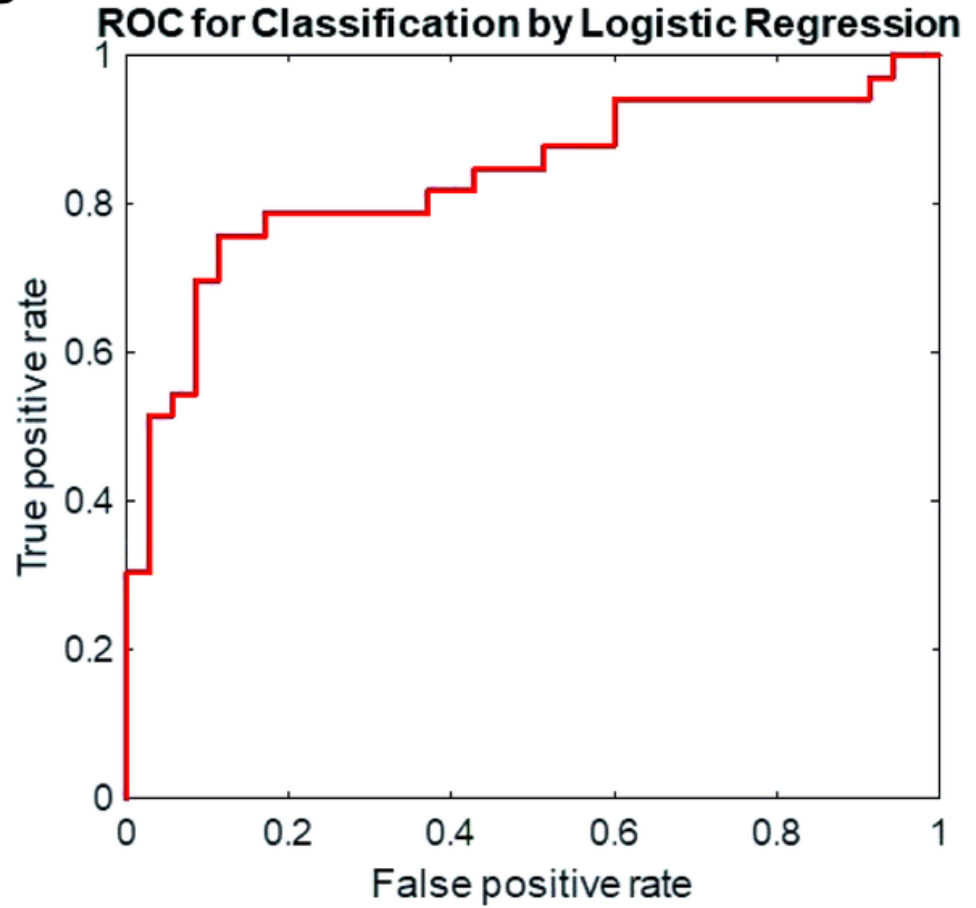

Figure 3

Prediction model of the persistence of pulmonary fibrosis. A. Identified 4 Parameters those can distinguish between two groups. B. The confusion matrix of the five-fold cross validation which was used to validate the performance of the model. C. Receiver operating characteristic curve which was used to evaluate accuracy, Positive Predictive Value (PPV), Negative Predictive Value (NPV) and Sensitivity and Specificity of the model. True positive rate $=$ Sensitivity; True negative rate = Specificity . 